"This is the peer reviewed version of the following article: Nutritional Care of Elderly Patients in Acute Care Settings: A Qualitative Study.,J ournal of Applied Gerontology 16(3):374-379 Mar 2016 which has been published in final form at https://doi.org/10.1111/ggi.12532 This article may be used for non-commercial purposes in accordance with Wiley Terms and Conditions for Self-Archiving." 


\section{Nutritional Care of Elderly Patients in Acute Care Settings:}

\section{A Qualitative Study}

Running title: Nutritional Care of Elderly Patients

Leila Gholizadeh, PhD, MSc, BSc

Lecturer, Faculty of Health

University of Technology, Sydney, Australia

Email: Leila.gholizadeh@uts.edu.au

Nahid Dehghan Nayeri, PhD, MSc, BSc

Associate Professor, Faculty of Nursing \& Midwifery

Tehran University of Medical Science, Iran

Email: nahid.nayeri@gmail.com

Eesa Mohammadi, PhD, MSc, BSc

Associate professor, Faculty of Medical Sciences

Tarbiat Modares University, Iran

Email: mohamade@modares.ac.ir

Khadijeh Yazdi, (PhD Candidate), MSc, BSc

Faculty of Nursing \& Midwifery

Tehran University of Medical Science, Iran

Email: yazdikh@yahoo.com

Corresponding Author:

Leila Gholizadeh

Faculty of Health

University of Technology, Sydney

PO Box 123, Broadway NSW 2007

Australia

Phone: + 61295144814

Email: Leila.gholizadeh@uts.edu.au 


\section{ABSTRACT}

Elderly patients are at increased risk of malnutrition. This study aimed to explore elderly patients' nutritional care in acute care settings from the perspectives of 12 nurses using individual interviews. Data were analysed according to the method of interpretive description. Aanalysis of data resulted in the emergence of two themes and 6 subthemes. The identified themes were: a) the importance of nutritional care for elderly patients is greatly acknowledged, but not necessarily provided' and b) 'nutritional care is not a priority for the health care system'. These themes and related subthemes suggest the need for improving elderly patients’ nutritional care. Patient nutrition care should be emphasized, as an integral part of holistic patient care, in the education of health professionals and be promoted through the provision of managerial support, allocating adequate budget, multi-disciplinary collaborative work and implementing an appropriate monitoring and evaluation system which allow quality assessment of nutritional care in patients.

Key words: Nutritional care, patient nursing care, elderly care 
As the elderly population is increasing globally, adjustments in health care provisions become necessary to meet the special needs of elderly consumers. Some $60 \%$ of hospital beds in medical surgical wards and $46 \%$ in intensive care units are occupied by elderly patients, with above $40 \%$ of the patients suffering from hospital associated complications, such as infection, pressure sore, and malnutrition (Chen, Yu, Song, \& Chui, 2010). General weakness, functional disability, and impaired level of consciousness are common risk factors in the elderly, resulting in many older people becoming dependent on others for meeting their basic day-to-day needs (Fitzpatrick, 2000; Westergren, Unosson, Ohlsson, Lorefält, \& Hallberg, 2002). These conditions, in conjunction with disease related-disabilities and hospital-associated complications such as malnutrition debilitate elderly patients. Evidence shows that $20-60 \%$ of patients suffer from malnutrition, leading to increased length of hospital stay, healthcare costs, and mortality and morbidity.

While an important factor in patient outcomes, malnutrition remains largely underrecognized in acute care settings (Compan, di Castri, Plaze, \& Arnaud-Battandier, 1999). Similar to many health care systems, malnutrition is a common problem in Iran, with one particular study revealing that $52 \%$ of patients admitted to a general hospital in Tehran was suffering from malnutrition (Hosseinpour-Niazi, Naderi, Delshad, \& Mirmiran, 2011).

Adequate nutritional intake is a basic human right and the cornerstone of good health (Naithani, Thomas, Whelan, Morgan, \& Gulliford, 2009). Current standards recommend that the nutritional needs of patients should be met through healthy and balanced diet and sufficient food and fluid intake encouraged by providing a comfortable and pleasant dining environment (Sloane, Ivey, Helton, Barrick, \& Cerna, 2008). Maintaining and promoting patient nutrition is an important aspect of holistic nursing care, which can improve patient 
health outcomes (Wentzel Persenius, Wilde-Larsson, \& Hall-Lord, 2009). Nutritional care should particularly be a care priority in elderly patients due to its significant impact on the patients' health status and ability to recover from diseases (Izawa et al., 2006).

Despite its importance, evidence shows that there is gap between the recommended nutritional care and actual practice in clinical settings (Wentzel Persenius et al., 2009). Patient nutrition is a neglected care arena in many health care services (Zeitz et al., 2011). Accordingly, this aspect of patient care has not been a focus of research and practice in Iran. Understanding the current practice and factors that can affect the provision of quality nutritional care to elderly patients is important to inform geriatric care practices. Certainly, nurses are in unique positions to intervene at multiple levels to promote patient nutrition care. This study aimed to explore the nature of nutritional care provision to elderly patients in acute care settings from the perspectives of nurses.

\section{METHODS}

This qualitative study was part of a larger project which aimed to explore the notion of nursing care provision to elderly patients in acute care facilities in Iran. The results of this study should be useful in informing the recent initiatives to improve specialized elderly care in this country. The study received approvals from the ethics committees of the participating university and hospital. The researcher explained the study aims and objectives to participants who volunteered to participate in the study and assured them of the confidentiality of their responses.

Using the purposive sampling method, 12 clinical nurses who had experience in caring for elderly patients were recruited from a tertiary general hospital in Tehran and participated in an individual semi-structured interviews. The interview times were scheduled at participants' convenience; all carried out at the hospital, and took an average 40 minutes to 
complete. An interview guide was used to provide a loose structure to the interviews. Each interview process began with a general open-ended question and followed by further questions based on the context of the participant's answer. The main interview question was: “tell me how nutritional care is provided to elderly patients in this hospital?'. Observations and field notes were also taken to supplement the interview data. The observations were made during four different occasions and arranged in different working shifts, each observation lasting between 60-150 minutes. Data collection continued until data saturation was achieved.

\section{Data analysis}

The interviews were recorded and transcribed verbatim by the principle researcher and data analysis began immediately after the first interview. An iterative coding process was applied, including comparing and contrasting data within and between individuals, comparing data to emerging codes and generating new codes. This approach allowed the emerging themes to be further explored in the subsequent interviews, reffered in the literature as the constant comparative method (Miles \& Huberman, 1994). Data were analyzed using the interpretive description method (S. Thorne, Kirkham, \& MacDonald-Emes, 1997; Sally. Thorne, Kirkham, \& O'Flynn-Magee, 2004), which enabled the researcher to describe, interpret, and develop a comprehensive understanding of the nature of nutritional care provided to elderly patients. Studies that use this approach have usually small sample sizes and employ various data collection methods, typically interview, observation, and field notes (S. Thorne et al., 1997; Sally. Thorne et al., 2004). A variety of strategies were used to enhance the rigor of study findings, including developing a close relationship between the researcher and participants, debriefing at the end of each interview, field observation, data saturation, data 
triangulation, member checking of transcripts (researcher triangulation), direct quotes, methodological information, and analytical decisions (Miles \& Huberman, 1994).

\section{RESULTS}

The analysis of the qualitative data revealed two themes and 6 sub-themes. The first theme, the importance of nutritional care for elderly patients is greatly acknowledged, but not necessarily provided, consisted of two sub-themes: 'nutritional care of elderly patients needs to be promoted ' and ' nutritional care means a lot to the elderly. The second theme, nutritional care is not a priority for the health care system, consisted of four sub-themes: 'unsupportive organizational environment', 'nutritional care is provided based on humanitarian considerations and not a commitment to nursing standards', 'nutritional care of elderly patients remains mainly the family's responsibility', and 'enhancing the dietary management of elderly patients requires collaborative care. The study themes and accompanying sub-themes are described in the following section.

\section{a) The importance of nutritional care for elderly patients is greatly acknowledged, but not necessarily provided}

This core study theme included the following sub-themes: 'Elderly patients' nutritional care needs to be enhanced' and 'nutritional care means a lot to the elderly', which are described accordingly.

\section{Elderly patients' nutritional care needs to be enhanced}

The nutritional nursing care for elderly patients revolved around patient assessment, physical support, education, and encouragement. Nurses commented on the significance of adopting a holistic approach to the elderly care including the need for nutritionally balanced diet. A rich and nutritious diet was perceived to be vital in maintaining the optimal health in elderly 
patients and boosting up patient recovery. Nutritional care should incorporate knowledge and skills of nursing staff of the dietary needs and requirements of the elderly, their food preferences and resource availability. Commenting on the importance of nutritional care in elderly patients, one nurse said:

We should provide better nutritional care to elderly patients; a rich nutritional diet is essential in patient recovery (N3).

Nurses commented on their experiences of providing nutritional care to elderly patients, stating that nutritional care begins with an assessment of patient's ability to eat independently. Assistance, education and encouragement are provided when required. The following is an excerpt from a nurse:

I should know that if an elderly patient does not eat her meal properly, she will be at risk... It is not enough just to leave the food on the table... I have to break the barriers... I must feed the patient myself (N5).

Nurses believed that elderly patients should be empowered through providing support and encouragement to take more responsibilities in their health care. Accordingly, patients should be supported to self-feed themselves and receive aid when needed. The followings are excerpts from two nurses:

I help them with holding the glass when drinking water to avoid splashing it on their clothes. At the meal time I provide them with tissue and raise head of the bed (N8).

You have to keep reminding them 'hold the spoon like this; [you have to] encourage them to eat adequate food (N4).

Nurses, however, expressed their dissatisfaction with the level of nutritional care provided to elderly patients in acute care settings. The current food service was widely criticized for being too inflexible with meal times, food options, and serving the patients with monotonous and hard to chew and digest food. The followings are excerpts from participating nurses: 
Food is repetitive: rice, stew and a bit soup every day. We should provide better nutrition for patients... Most elderlies do not like the repetitive breakfast of butter, cheese, and jam, the same day after day. They may like to have some porridge with a glass of fresh milk for breakfast, but these types of foods are not served in our hospital. How can we expect the patients to have timely recovery despite such poor nutritional care? ...Dinner is served at 18:00 sharp; no access to food after that. Patients have to drink tea till morning (N8).

Some types of foods served here (in the hospital), patients say they never cook and eat such foods at home... The patient says I cannot eat this food; I cannot swallow them; I eat different food at home. I like soup, soft foods (N3).

\section{Nutritional care means a lot to the elderly}

Nurses believed that promoting good nutrition is important not only for maintaining physical health it also has positive psychological effects and entails respect for elderly patients' dignity. Food and mealtimes are a high priority for older people and enjoyable mealtimes can improve their health and wellbeing. Taking into consideration elderly patients' food preferences, eating styles and providing them with timely assistance are important aspects of respecting the dignity of elderly patients. Followings are excerpts from participating nurses:

Patients should have access to fresh milk or a drink at all times. This is important not only from nutritional point of view, also patients feel better psychologically (N5).

We should be careful not to spill the food on their clothes when feeding them...Some elderlies are very sensitive and get offended easily... Sometimes I sit by them and help them with their food ... They expect us to attend to their needs immediately, otherwise they get offended... They act like children (N9).

\section{b) Nutritional care is not a priority for the health care system}

The second theme that emerged from data indicated that in the current health care system patient nutritional care is not receiving adequate attention. This core theme was consisted of 
four sub-themes: 'unsupportive organizational environment', 'nutritional care is provided based on humanitarian considerations and not a commitment to nursing standards', 'nutritional care of unable elderly patients remains mainly the family’s responsibility', and 'enhancing the dietary management of elderly patients requires collaborative care'. These subthemes are explained further below.

\section{Unsupportive organizational environment}

Unsupportive organizational environment was emerged as a strong theme, which was perceived as having adverse effect on the provision of nutritional care to elderly patients. Some organizational factors such as inadequate quantity and quality of the hospital food interfered with the nurses' desire to promote nutritional care for patients. Nurses highlighted some service shortages indicating that patient nutrition care often failed to receive priority attention in the clinical practice. In view of that, there were lack of appropriate monitoring and evaluation systems in place to identify the shortcomings in patient nutritional care, contributing to the poor food service.

A nurse manager said:

We have elderly patients who are alone (without a family caregiver), nobody comes to visit them; nobody clean them and nobody help them with their food. A novice nurse gives their medications. Well, the patient's medications have been given and documentation is complete. Everything looks OK legally; but nobody monitors their food intake (N6).

\section{Nutritional care is provided based on humanitarian considerations and not a commitment to nursing standards}

Lack of time was the most often mentioned barrier to the provision of nutrition care in the hospital setting. It was commented that the managers; and thus, nurses placed high priority to nursing procedures, such as dressings, administering patient medications, and attending to doctors' orders. In the view of nutrition care not being a priority for the health system and the 
lack of monitoring and evaluation systems in place, nurses who were involved in providing nutritional care to elderly patients, were rather motivated by sympathy than viewing patient nutrition care as part of professional nursing practice. An excerpt from two participating nurses indicates this.

They (elderly patients) are helpless and rely on us. I feel sorry for them; so despite my heavy workload, I try to help them at mealtimes (N2).

When caring for older patients you have to consider humanity. You know, there is not enough recognition for the work you do. Some nurses say there is no salary difference between a dodgy nurse and a nurse who puts her heart and soul into the work (N1).

\section{Nutritional care of elderly patients remains mainly the family's responsibility}

Patients' families were often involved in providing nutritional care to their elderly patients. Nurses encouraged the family to bring the patient's favorite food from home and provide them with between-meal snacks. The following is an excerpt from a nurse working in an internal ward:

We advise families to bring over the patient's favorite food... foods served in the hospital are hard to digest... they are monotonous and poorly balanced (N3).

Family members also became involved in helping the patients at mealtimes. Due to nursing shortages and the increased workload, the involvement of the family in elderly patient care seemed imperative. Patient nutrition care was compromised at times when a family member was not at the hospital. A nurse said:

Sometimes in the handover, I ask why this patient's food is left on the table; they say her family member wasn't here to feed her (N6).

While the importance of high quality food intake for elderly patients was clearly understood by nurses, this aspect of patient care was easily overlooked due to the heavy nursing workload. The below is an excerpt from a nurse: 
We are Muslim!, but I am sorry I have to say that we often even don’t have enough time to properly check patients’ vital signs. It is a shame to be a nurse and watch patients’ food left untouched on the tables (N7).

\section{Enhancing the dietary management of elderly patients requires collaborative care}

Effective nutritional care was perceived requires the collaboration of different sectors of the hospital, including the management, finance, kitchen, and nursing. Nurses believed that changes for promoting patient nutrition care should be started from managerial level. Allocating adequate food budget, staff education, and putting in place an appropriate assessment and monitoring system could help improve the quality of nutritional care to patients, in particular elderlies. The following is an excerpt from a head-nurse:

Sometimes I tell a nursing staff, let's go and help out the patient with his food. As soon as I intervene, they (staff) complete the required care themselves (N1).

Appropriate staffing and the use of combined experiences in providing care to elderly patients can contribute to the improvements in patient nutrition care. A participating headnurse said:

Sometimes, novice nurses are rostered in a working shift. It is more common you see untouched foods on the tables in such shifts. Novice nurses are not usually keen to feed patients. Experienced nurses are rather more involved in patient nutritional care; at least they get somebody to do it. When elderly patients are hospitalized in a ward, the presence of an experienced nurse in each working shift is very important. This needs the cooperation of the chief nursing officer (who completes the roster) (N1).

\section{DISCUSSION}

This study addressed the nutritional care of elderly patients in an acute care setting in Iran. In the view of the increased risk of malnutrition in elderly population, a high percentage of elderly patients are undernourished when admitted to hospital and malnutrition develops further in majority of these patients while in hospital (Kondrup, Allison, Elia, Vellas, \& 
Plauth, 2003). Historically, patient nutrition care has not gained adequate clinical and research attention, particularly in developing countries including Iran. Increasingly; however, there is more attention being given to patient nutrition in developed health care systems (Miles \& Huberman, 1994). Given the fact that malnutrition is more common in elderly patients in Iran (Hosseinpour-Niazi et al., 2011), understanding the nature of nutritional care provided to the patients in the course of their admission to health care facilities is important and provides evidence to support the need for improved nutritional care to elderly patients. Additionally, new initiatives are being undertaken by the Iranian Ministry of Health to improve elderly care in the country through supporting the specialty studies of elderly care; thus, exploring the current practice in elderly patients' care is a timely research topic and helps inform the development of educational curriculums for the geriatric care specialties.

The findings of this study showed that the significance of nutrition for elderly patients was highly understood by nurses. There were considerable comments on the important role of good nutrition in elderly patients' health and recovery. Nurses became involved in the nutritional care of elderly patients through providing the patients with physical support, education and encouragement. The aim of the nutritional nursing care was to encourage the self-care abilities of elderly patients to eat their meal independently and receive assistance when they need it. While working with limited resources, nurses made efforts to enhance nutritional care for elderly patients. They involved the patient's family in providing nutrition care to the elderly to recompense for the nursing shortages. The family's involvement included providing physical assistance to the patient at mealtimes and bringing over quality and favorable homemade food. Although engaging the family in the care of hospitalized patients, particularly activities of daily living has been documented in the literature (Godfrey, Cloete, Dymond, \& Long, 2012) and can be employed to improve nutritional care of elderly patients, families’ desire for becoming involved in patient care should be discussed and 
appropriate guidance and supervision provided (Halm \& Titler, 1990). More importantly, the family’s involvement in patient nutrient care should not shirk nurses’ responsibility for providing this aspect of patient care, as it was observed in the present study. That is, in some occasions patients' foods were left untouched on the tables in the obscene of their family member.

In this study, the cultural tradition of respecting the elderly in Iran was emerged as a supporting factor in the provision of elderly care. Nurses described helping an elderly patient with their food as a satisfying and nourishing experience. Some nurses felt disgruntled about not being able to provide adequate assistance to elderly patients. Religion was also appeared as a supporting factor to the elderly care, in that some nurses who were not able to provide the elderly with optimal care expressed a sense of guilt and not being deserved to be called as "Muslim”. Respecting for elderly people and caring for ill patients are highly emphasized in Islam (Queensland Health and Islamic Council of Queensland., 2010). These enabling cultural factors need to be further identified and applied for patient care promotion.

In current study, the health care system was widely criticized for not placing adequate priority on patient nutrition care. Lack of time due to nursing shortages and the heavy workload was frequently mentioned as the barrier to appropriate elderly care, including patient nutritional care. Nurses expressed their lack of satisfaction with the level of nutritional care provided to elderly patients and they highlighted the need for promoting this arena of care. These findings support the results of a study conducted by Nasiri et al (2007) in Iran, reporting that $58 \%$ of hospitalized patients were not satisfied with the quality of food services in hospitals and 49.2\% expressed dissatisfaction with the available food options. Additionally, $60.7 \%$ of the patients reported that their need for a special diet was not met in hospital (Hosseinpour-Niazi et al., 2011). 
Nurses believed that for elderly patients, food is highly valued not only for its role in patient health and recovery, but also elderly patients regarded nutritional care as emotionally satisfying, conveying 'respect' and 'caring' for the patients. The importance of paying attention to individual needs of elderly patients has been highlighted in the literature. Anderberg et al. (2007) suggested that implementation of an individual care plan is important to maintain the dignity of the elderly and meet the patients individual care needs (Anderberg, Lepp, Berglund, \& Segesten, 2007).

In the view of lack of an appropriate monitoring and evaluation system for nutritional care, nurses perceived nutritional care of elderly patients as a compassionate and humanistic care, leading to a sense of satisfaction in nurses and a sense of dignity in elderly patients. Similarly, in a grounded theory study, Wentzel Persenius et al. (2009) found that the balance between individual and routine care generates a sense of satisfaction and achievement in nurses (Wentzel Persenius et al., 2009). Nevertheless, patient nutritional care should be emphasized as an important part of holistic nursing in nursing curriculums and a minimum set of standards adopted (Godfrey et al., 2012). Well trained and supported health care providers are the key to ensuring that the quality standards in nutritional care are met (the BAPEN Quality Group., 2010).

It was commonly acknowledged that enhancing the dietary management of elderly patients required collaborative multidisciplinary care, a finding that supports the results of past research (The Royal College of Nursing., 2010). It is essential that members of the multidisciplinary team understand the importance of good nutritional care in the elderly and their role in providing it and take responsibility in promoting this area of patient care through effective collaboration with the patient, colleagues, and the patient's family (the BAPEN Quality Group., 2010). There is evidence that effective management of malnutrition helps reduce the burden on health and care resources (Arnaud Battandier et al., 2004). 
Yet, administrative support from the organisation is essential to enhance service provision (The Royal College of Nursing., 2010). In their study, Copeman et al. (2000) found that organizational factors such as poor managerial structure and lack of appropriate staff education were related to patient malnutrition (Copeman, 2000). Elderly patients are at risk of malnutrition due to factors, such as loss of taste and factors associated to their disease and treatment, such as chronic pain and loss of appetite. Poor food-serving services and environment exacerbate the situation [18]. Nurses in current study believed that the main changes need to be initiated from the management in a top to bottom approach. Developing and implementing policies that place priorities on patient nutritional care and providing supportive organizational environment are necessary primary activities. In a routine-oriented practice, nurses usually adhere to some predetermined obligated routines and they fail to meet the individual care needs of patients, such as their need for assistance during mealtime (Molazem, Ahmadi, Mohammadi, \& Bolandparvaz, 2011). Expectedly, nutritional care is more likely to be compromised in a health care system where missing a patient's meal is not legally as important and prosecutable as missing a medical treatment (the BAPEN Quality Group., 2010). As suggested by participating nurses in this study, a good balance of new and experienced nursing staff in each working shift might help improve the nutritional care of elderly patients.

Nurses believed that there should be an appropriate system of assessment and evaluation of patient nutritional care, a finding that has been highlighted in the literature [14]. In an attempt to promote patient nutrition, the British Association for Parenteral and Enteral Nutrition (BAPEN, 2010) recommended nutritional screening for all patients and ensuring that vulnerable patients receive appropriate nutritional care and the practical assistance they need at mealtimes (the BAPEN Quality Group., 2010). Using a nutritional assessment tool 
can help identifying patients who are at risk of malnutrition, such as the Mini Nutritional Assessment (MNA) which as has been designed and validated to provide a rapid assessment of nutritional status in elderly patients in health care facilities (Vellas et al., 1999). Other malnutrition assessment tools, such as MUST (the Malnutrition Universal Screening Tool) which has been developed by the BUPEN can also guide appropriate actions. However, it is important that the elderly patient remains the focus of care.

\section{CONCLUSION}

The findings of this study suggest the need for improving nutritional care for elderly patients. Patient nutrition care should be emphasized, as an integral part of holistic patient care, in the education of health professionals and be promoted through the provision of managerial support, allocating adequate budget, multi-disciplinary collaborative work and implementing an appropriate monitoring and evaluation system which allow quality assessment of nutritional care in patients. 


\section{REFERENCES}

Anderberg, Patrice, Lepp, Margret, Berglund, Anna-Lena, \& Segesten, Kerstin. (2007). Preserving dignity in caring for older adults: a concept analysis. Journal of Advanced Nursing, 59(6), 635643. doi: 10.1111/j.1365-2648.2007.04375.x

Arnaud Battandier, F., Malvy, D., Jeandel, C., Schmitt, C., Aussage, P., Beaufrere, B., \& Cynober, L. (2004). Use of oral supplements in malnourished elderly patients living in the community: a pharmaco-economic study. Clinical Nutrition, 23(5), 1096-1103.

Chen, Z., Yu, J., Song, Y., \& Chui, D. . (2010). Aging Beijing: Challenges and strategies of health care for the elderly. Ageing research reviews, 9, S2-S5.

Compan, B., di Castri, A., Plaze, J. M. , \& Arnaud-Battandier, F. (1999). Epidemiological study of malnutrition in elderly patients in acute, sub-acute and long-term care using. The Journal of Nutrition, Health \& Aging, 3(3), 146-151.

Copeman, J. (2000). Promoting nutrition in older people in nursing and residential homes. British Journal of Community Nursing, 5(6), 277-278.

Fitzpatrick, Joanne. (2000). Oral health care needs of dependent older people: responsibilities of nurses and care staff. Journal of Advanced Nursing, 32(6), 1325-1332. doi: 10.1046/j.13652648.2000.01631.x

Godfrey, H., Cloete, J., Dymond, E., \& Long, A. (2012). An exploration of the hydration care of older people: a qualitative study. International Journal of Nursing Studies 49(10), 1200-1211.

Halm, Margo A., \& Titler, Marita G. (1990). Appropriateness of critical care visitation: Perceptions of patients, families, nurses, and physicians. Journal of Nursing Care Quality, 5(1), 25-37.

Hosseinpour-Niazi, S., Naderi, Z., Delshad, M., \& Mirmiran, P. (2011). Prevalence of malnutrition in hospitalized patients in Taleghani Hospital in Tehran. Journal of Gorgan University of Medical Sciences, 13(4), 97-106. 
Izawa, Sachiko, Kuzuya, Masafumi, Okada, Kiwako, Enoki, Hiromi, Koike, Teruhiko, Kanda, Shigeru, \& Iguchi, Akihisa. (2006). The nutritional status of frail elderly with care needs according to the mini-nutritional assessment. Clinical nutrition (Edinburgh, Scotland), 25(6), 962-967.

Kondrup, J., Allison, S. P., Elia, M., Vellas, B., \& Plauth, M. (2003). ESPEN Guidelines for Nutrition Screening 2002. Clinical Nutrition, 22(4), 415-421. doi: http://dx.doi.org/10.1016/S0261$\underline{5614(03) 00098-0}$

Miles, M. B., \& Huberman, A. M. (1994). Qualittaive data analysis (2nd ed.). Thousand Oaks: SAGE publications.

Molazem, Z., Ahmadi, F., Mohammadi, E., \& Bolandparvaz, S. (2011). Improvement in the nursing care quality in general surgery wards: Iranian nurses' perceptions. Scandinavian Journal of Caring Sciences, 25(2), 350-356. doi: 10.1111/j.1471-6712.2010.00833.x

Naithani, Smriti, Thomas, Jane E., Whelan, Kevin, Morgan, Myfanwy, \& Gulliford, Martin C. (2009). Experiences of food access in hospital. A new questionnaire measure. Clinical nutrition (Edinburgh, Scotland), 28(6), 625-630.

Queensland Health and Islamic Council of Queensland. (2010). Health care providers' handbook on Muslim patients (2nd ed.): Queensland Health: Division of the Chief Health Officer.

Sloane, Philip D., Ivey, Jena, Helton, Margaret, Barrick, Ann Louise, \& Cerna, Ana. (2008). Nutritional Issues in Long-Term Care. Journal of the American Medical Directors Association, 9(7), 476485. doi: http://dx.doi.org/10.1016/i.jamda.2008.03.005

the BAPEN Quality Group. (2010). Malnutrition matters: Meeting quality standards in nutritional care. Retrieved June 08, 2013, from http://www.bapen.org.uk/pdfs/toolkit-forcommissioners.pdf

The Royal College of Nursing. (2010). Enhance nutritional care bookl. from http://www.rcn.org.uk/development/practice/nutrition/enhancing nutritional care 
Thorne, S., Kirkham, S. R., \& MacDonald-Emes, J. . (1997). Focus on qualitative methods. Interpretive description: a noncategorical qualitative alternative for developing nursing knowledge. Research in Nursing \& Health, 20(2), 169-177.

Thorne, Sally., Kirkham, S. R., \& O'Flynn-Magee, K. (2004). The analytic challenge in interpretive description. International Journal of Qualitative Methods, 1(3).

Vellas, Bruno, Guigoz, Yves, Garry, Philip J., Nourhashemi, Fati, Bennahum, David, Lauque, Sylvie, \& Albarede, Jean-Louis. (1999). The mini nutritional assessment (MNA) and its use in grading the nutritional state of elderly patients. Nutrition, 15(2), 116-122. doi: http://dx.doi.org/10.1016/S0899-9007(98)00171-3

Wentzel Persenius, M., Wilde-Larsson, B., \& Hall-Lord, M. L. (2009). To have and to hold nutritional control: balancing between individual and routine care. A grounded theory study. Intensive \& critical care nursing : the official journal of the British Association of Critical Care Nurses, 25(3), 155-162.

Westergren, A., Unosson, M., Ohlsson, O., Lorefält, B., \& Hallberg, I. R. (2002). Eating difficulties, assisted eating and nutritional status in elderly ( $>$ or $=65$ years) patients in hospital rehabilitation. International Journal of Nursing Studies, 39(3), 341-351.

Zeitz, Kathryn, Kitson, Alison, Gibb, Heather, Bagley, Eliza, Chester, Margaret, Davy, Cathy, ... . Shanks, Alison. (2011). Working together to improve the care of older people: a new framework for collaboration. Journal of Advanced Nursing, 67(1), 43-55. doi: 10.1111/j.13652648.2010.05478.x 